\title{
Two-dimensional dynamics of a free molecular chain with a secondary structure
}

\section{Zolotaryuk, Alexander; Christiansen, Peter Leth; Savin, A.V.}

Published in:

Physical Review E. Statistical, Nonlinear, and Soft Matter Physics

Link to article, DOI:

10.1103/PhysRevE.54.3881

Publication date:

1996

Document Version

Publisher's PDF, also known as Version of record

Link back to DTU Orbit

Citation (APA):

Zolotaryuk, A., Christiansen, P. L., \& Savin, A. V. (1996). Two-dimensional dynamics of a free molecular chain with a secondary structure. Physical Review E. Statistical, Nonlinear, and Soft Matter Physics, 54(4), 3881-3894. https://doi.org/10.1103/PhysRevE.54.3881

\section{General rights}

Copyright and moral rights for the publications made accessible in the public portal are retained by the authors and/or other copyright owners and it is a condition of accessing publications that users recognise and abide by the legal requirements associated with these rights.

- Users may download and print one copy of any publication from the public portal for the purpose of private study or research.

- You may not further distribute the material or use it for any profit-making activity or commercial gain

- You may freely distribute the URL identifying the publication in the public portal 


\title{
Two-dimensional dynamics of a free molecular chain with a secondary structure
}

\author{
A. V. Zolotaryuk, ${ }^{1,2}$ P. L. Christiansen, ${ }^{1}$ and A. V. Savin ${ }^{3}$ \\ ${ }^{1}$ Institute of Mathematical Modelling, Technical University of Denmark, DK-2800 Lyngby, Denmark \\ ${ }^{2}$ Bogolyubov Institute for Theoretical Physics, 252143 Kyiv, Ukraine \\ ${ }^{3}$ Institute for Physico-Technological Problems, Prechistenka Street, 13/7, 119034 Moscow, Russia
}

(Received 11 April 1995; revised manuscript received 11 December 1995)

\begin{abstract}
A simple two-dimensional (2D) model of an isolated (free) molecular chain with primary and secondary structures has been suggested and investigated both analytically and numerically. This model can be considered as the simplest generalization of the well-known Fermi-Pasta-Ulam model of an anharmonic chain in order to include transverse degrees of freedom of the chain molecules. Both the structures are provided by the first- and second-neighbor intermolecular bonds, respectively, resulting in a regular zig-zag (" $2 D$ helix") chain on a plane. The set of two coupled nonlinear field equations with respect to the longitudinal and transverse displacements of the chain molecules has been derived. Two types of stable (nontopological) soliton solutions which describe either (i) a supersonic solitary wave of longitudinal stretching accompanied by transverse slendering or, as in the 1D model, (ii) supersonic pulses of longitudinal compression propagating together with localized transverse thickening (bulge) have been found. Some peculiar stability properties of these two-component soliton solutions have been discovered by using numerical techniques developed in this paper. [S1063-651X(96)10809-6]
\end{abstract}

PACS number(s): 68.45.Kg, 63.20.Ry, 63.20.Pw

\section{INTRODUCTION}

The 1D nonlinear (anharmonic) lattices, which have been studied in a series of papers (see, e.g., Refs. [1-6]), are usually considered as the basic models to describe transport properties in biomolecules. Any intermolecular potential in such a 1D lattice (chain) has a hardening (positive) anharmonicity. This is a conventional type of anharmonicity in nonlinear lattices and its physical meaning is as follows. When neighboring atoms (or molecules) of the chain are displaced from their equilibrium positions, the repulsion force between them becomes stronger than the harmonic approximation of this interaction. In other words, a hardening anharmonic force contributes to this repulsion with the positive sign. As a result of the presence of such a positive anharmonicity, dynamically stable solitary waves can propagate along the chain with supersonic velocities. These nonlinear collective excitations are referred to as the lattice (or acoustic) solitons. For some particular choices of the intermolecular potential, like Toda, Boussinesq, etc., the solitons are called accordingly.

After the pioneering work [7], where it was discovered that the solitonic excitations in 1D nonlinear lattices are extremely sensitive to their transverse perturbations, a series of papers (see, e.g., works [8-11] and references therein) has appeared on the studies of soliton propagation in nonlinear lattices when transverse motions of the chain molecules are taken into account. Moreover, in some cases the transverse displacements of molecules are considered as the most important motions in biophysical processes. Thus, in the DNA molecule, the stretching of the base-pairs in the transverse direction determines the fundamental mechanism of the denaturation of this molecule. The Peyrard-Bishop model of DNA melting [12-14] has just been formulated in terms of only the transverse motions of the two complementary strands. Although the DNA molecule is considered in this approach as an isolated object, the model actually describes the 1D dynamics of the chain molecules in an effective substrate (on-site) potential.

In this paper, we allow the chain molecules to move in both the longitudinal and perpendicular (transverse) directions and the chain is considered as an isolated (free) object which is not subjected to any substrate potential. Such a 2D molecular chain is supposed to have some regular structure when it is found in an undistorted (ground) state. Such a requirement immediately leads with necessity to some secondary structure and this is really the case in biology for many macromolecules (DNA, protein, etc.). Geometrically, the secondary structure is realized in the form of a helix.

The aim of the present paper is to study the soliton propagation in an isolated molecular chain for which (i) the transverse motions of the molecules and (ii) helical structure are taken into account. For simplicity of the analytical investigation we consider only one transverse dimension. As a result, the 3D helical structure is essentially simplified, transforming into the " $2 \mathrm{D}$ helix" which is merely a planar zig-zag chain. Then the primary and secondary structures are provided by the first- and second-neighbor intermolecular interactions, respectively. Such a system can be considered as the simplest theoretical model of an isolated molecular chain and it corresponds to the realistic situation in biomolecular sciences. More precisely, the planar zig-zag structure is much closer to the reality of biomolecular dynamics than the 1D nonlinear lattices. Note that on the qualitative level, the 2D and 3D cases should not essentially differ from each other. However, the difference between the 1D and 2D cases, as was expected and pointed out earlier [7-10], turns out to be drastic.

It should be emphasized that the important point of the zig-zag model is the secondary structure. Even in the 1D chain, the introduction of the second-neighbor interactions crucially changes the dynamics of the system $[15,16]$. There- 


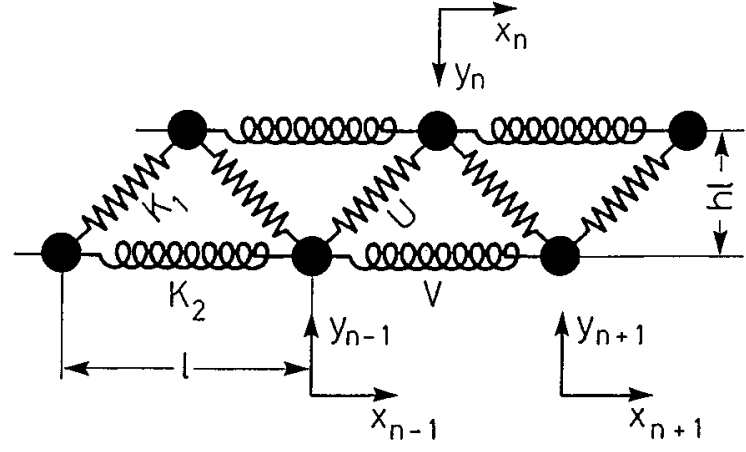

FIG. 1. Schematic representation of a zig-zag molecular chain $(h=1 / 2)$.

fore the zig-zag structure with the first- and second-neighbor interactions, which can be referred to as valence and hydrogen bonds, respectively, essentially make the theory more sophisticated. Note that even if the molecules are assumed to be coupled by harmonic forces, an effective anharmonicity appears because of the geometry of the system. For breatherlike solutions, the effects of such a "geometrical" nonlinearity have previously been investigated by Cadet [17].

The paper is organized as follows. In the following section we describe a planar zig-zag model with the first- and second-neighbor interactions. In this section we derive the basic equations of motion which have the differencedifferential form. The transition to the continuum limit is described in Sec. III and, as a result, two pairs of coupled partial differential equations have been derived. Each pair has the corresponding 1D analog, the ill-posed or improved form of the Boussinesq equation. The linear dispersion law for the motion of small-amplitude waves in the zig-zag chain is studied in Sec. IV. The next section is devoted to the analytical studies of solitary-wave solutions in the continuum limit. In Sec. VI we develop a numerical method for seeking localized solutions of a stationary profile which are taken in the next section as initial conditions for simulations of the equations of motion. The comparison of the initial and final soliton profiles has been carried out there. The concluding remarks on the results of the present paper are outlined in Sec. VIII.

\section{A ZIG-ZAG CHAIN MODEL}

Let molecules (e.g., amino acids) be linked together in a molecular (polypeptide) chain, as illustrated in Fig. 1, by the first- and second-neighbor forces with the stiffness constants $K_{1}$ and $K_{2}$, respectively. This chain has the 2D helical (i.e., planar zig-zag) structure and its molecules are allowed to move and rotate in the $\mathcal{X Y}$ plane. The chain backbone, with lattice spacing $l$, is directed along the $\mathcal{X}$ axis and it can be considered as consisting of two linear chains coupled by valence bonds. Let the molecules of this backbone be situated at sites $\mathcal{X}=n l$, with the integers $n=0, \pm 1, \ldots$ for one of the chains and the half-integers $n= \pm 1 / 2, \pm 3 / 2, \ldots$ for the other chain as shown in Fig. 1. The chain molecules are supposed to have two degrees of freedom: the longitudinal $\left(x_{n}\right)$ and transverse $\left(y_{n}\right)$ displacements from the equilibrium positions stabilized by the zig-zag structure. The dimension- less parameter $h$ describes the geometry of the chain, namely the thickness of the zig-zag structure. Then the equilibrium distance between each pair of the nearest-neighboring molecules is determined by the dimensionless parameter $b=\sqrt{h^{2}+1 / 4}$.

The total Hamiltonian of the planar zig-zag system is given by

$$
H=\sum_{n}\left\{\frac{1}{2} M\left(\dot{x}_{n}^{2}+\dot{y}_{n}^{2}\right)+K l^{2}\left[U\left(r_{n}\right)+V\left(q_{n}\right)\right]\right\},
$$

where $M$ is the mass of molecules (e.g., peptide groups) and $K$ is the characteristic stiffness constant. The dimensionless intermolecular potentials $U(r)$ and $V(q)$ describe the primary (between the nearest neighbors) and secondary (between the second neighbors) interactions, respectively. The couplings between the nearest-neighboring molecules can be referred to as valence bonds while the secondneighboring interactions can be called hydrogen bonds. According to Fig. 1, the (dimensionless) deviations of the equilibrium lengths are defined by

$$
\begin{aligned}
& r_{n}=\sqrt{\left(\frac{1}{2}+\frac{x_{n+1}-x_{n}}{l}\right)^{2}+\left(h-\frac{y_{n}+y_{n+1}}{l}\right)^{2}}-b \\
& q_{n}=\sqrt{\left(1+\frac{x_{n+1}-x_{n-1}}{l}\right)^{2}+\left(\frac{y_{n+1}-y_{n-1}}{l}\right)^{2}}-1 .
\end{aligned}
$$

The potentials $U(r)$ and $V(q)$ are normalized as follows:

$U(0)=0=V(0), \quad U^{\prime \prime}(0)=\kappa_{1}, \quad$ and $\quad V^{\prime \prime}(0)=\kappa_{2}$,

where the primes denote the differentiation of the functions $U(r)$ and $V(q)$ with respect to their arguments and $\kappa_{1}=K_{1} / K$ and $\kappa_{2}=K_{2} / K$ are the dimensionless stiffness constants of the primary and secondary interactions, respectively.

The corresponding Euler-Lagrange equations of motion are

$$
\begin{aligned}
M \ddot{x}_{n} & =-K l^{2} \frac{\partial}{\partial x_{n}} W_{n} \text { and } M \ddot{y}_{n}=-K l^{2} \frac{\partial}{\partial y_{n}} W_{n}, \\
W_{n} & =U\left(r_{n-1}\right)+U\left(r_{n}\right)+V\left(q_{n-1}\right)+V\left(q_{n+1}\right) .
\end{aligned}
$$

For the dimensionless description it is convenient to introduce the dimensionless time

$$
\tau=\omega_{0} t, \quad \omega_{0}=\sqrt{K / M} .
$$

The corresponding lattice fields can also be rescaled to the dimensionless form as $u_{n}(\tau)=x_{n}(t) / l$ and $v_{n}(\tau)=y_{n}(t) / l$.

It follows from the form of the deviations of the equilibrium distances $r_{n}$ and $q_{n}$ given by Eqs. (2) that the dynamical equations (4) can be rewritten in terms of the lattice fields

$$
\rho_{n}=u_{n+1}-u_{n} \text { and } \eta_{n}=v_{n}+v_{n+1} \text {. }
$$

Indeed, keeping the same notations for the deviations $r_{n}(\tau)$ and $q_{n}(\tau)$ defined as functions of $\tau$, we obtain 


$$
\begin{gathered}
r_{n}=\sqrt{\left(\frac{1}{2}+\rho_{n}\right)^{2}+\left(h-\eta_{n}\right)^{2}}-b, \\
q_{n}=\sqrt{\left(1+\rho_{n-1}+\rho_{n}\right)^{2}+\left(\eta_{n}-\eta_{n-1}\right)^{2}}-1 .
\end{gathered}
$$

Next, it is convenient to introduce the following abbreviations:

$$
\begin{aligned}
& P_{n}=\frac{\partial}{\partial \rho_{n}} U\left(r_{n}\right), \quad Q_{n}=\frac{\partial}{\partial \rho_{n}} V\left(q_{n}\right), \\
& S_{n}=\frac{\partial}{\partial \eta_{n}} U\left(r_{n}\right), \quad T_{n}=\frac{\partial}{\partial \eta_{n}} V\left(q_{n}\right) .
\end{aligned}
$$

Then Eqs. (4) are transformed to the dimensionless form as follows:

$$
\begin{gathered}
\frac{d^{2} \rho_{n}}{d \tau^{2}}=P_{n+1}-2 P_{n}+P_{n-1}+Q_{n+2}-Q_{n+1}-Q_{n}+Q_{n-1} \\
\frac{d^{2} \eta_{n}}{d \tau^{2}}=-\left(S_{n-1}+2 S_{n}+S_{n+1}\right)+T_{n+1}-T_{n-1}+T_{n+2}-T_{n} .
\end{gathered}
$$

This "canonical" representation of the equations of motion is preferable for localized solutions when the relative displacement field $\rho_{n}(\tau)$ rapidly decreases to zero at infinity.

\section{THE CONTINUUM LIMIT}

In order to find an adequate continuum version of the basic difference-differential equations (9) and (10), we introduce in this section the following six generating functions of two real continuous variables $z_{1}$ and $z_{2}$. The first two of these functions come from the definition of the distance deviations defined by Eqs. (7):

$$
\begin{gathered}
r\left(z_{1}, z_{2}\right)=\sqrt{\left(\frac{1}{2}+z_{1}\right)^{2}+\left(h-z_{2}\right)^{2}}-b, \\
q\left(z_{1}, z_{2}\right)=\sqrt{\left(1+z_{1}\right)^{2}+z_{2}^{2}}-1 .
\end{gathered}
$$

Therefore, Eqs. (7) can be rewritten in terms of these functions as follows:

$$
\begin{aligned}
r_{n}= & r\left(z_{1}=\rho_{n}, z_{2}=\eta_{n}\right) \\
& \text { and } q_{n}=q\left(z_{1}=\rho_{n-1}+\rho_{n}, z_{2}=\eta_{n}-\eta_{n-1}\right) .
\end{aligned}
$$

The remaining four generating functions are defined by

$$
\begin{aligned}
& P\left(z_{1}, z_{2}\right)=\frac{\partial}{\partial z_{1}} U\left[r\left(z_{1}, z_{2}\right)\right]=\frac{1 / 2+z_{1}}{b+r\left(z_{1}, z_{2}\right)} U^{\prime}\left[r\left(z_{1}, z_{2}\right)\right], \\
& Q\left(z_{1}, z_{2}\right)=\frac{\partial}{\partial z_{1}} V\left[q\left(z_{1}, z_{2}\right)\right]=\frac{1+z_{1}}{1+q\left(z_{1}, z_{2}\right)} V^{\prime}\left[q\left(z_{1}, z_{2}\right)\right],
\end{aligned}
$$

$$
\begin{aligned}
& S\left(z_{1}, z_{2}\right)=\frac{\partial}{\partial z_{2}} U\left[r\left(z_{1}, z_{2}\right)\right]=\frac{z_{2}-h}{b+r\left(z_{1}, z_{2}\right)} U^{\prime}\left[r\left(z_{1}, z_{2}\right)\right], \\
& T\left(z_{1}, z_{2}\right)=\frac{\partial}{\partial z_{2}} V\left[q\left(z_{1}, z_{2}\right)\right]=\frac{z_{2}}{1+q\left(z_{1}, z_{2}\right)} V^{\prime}\left[q\left(z_{1}, z_{2}\right)\right],
\end{aligned}
$$

where the primes, as before, denote the differentiation of the functions $U(r)$ and $V(q)$ with respect to their arguments $r$ and $q$, respectively. Similarly, the set of the lattice fields (8) can be represented as the appropriate values of the functions (13):

$$
\begin{gathered}
P_{n}=P\left(z_{1}=\rho_{n}, z_{2}=\eta_{n}\right), \\
Q_{n}=Q\left(z_{1}=\rho_{n-1}+\rho_{n}, z_{2}=\eta_{n}-\eta_{n-1}\right), \\
S_{n}=S\left(z_{1}=\rho_{n}, z_{2}=\eta_{n}\right), \\
T_{n}=T\left(z_{1}=\rho_{n-1}+\rho_{n}, z_{2}=\eta_{n}-\eta_{n-1}\right) .
\end{gathered}
$$

The next step in the transition to the continuum limit, when $n=\mathcal{X} / l \equiv x$, is to expand correctly the spatial differences in Eqs. (9) and (10). These expansions can be represented in terms of the generating functions (13). Indeed, replacing the discrete fields $r_{n}(\tau)$ and $q_{n}(\tau)$ by the continuous functions $r(x, \tau)$ and $q(x, \tau)$, respectively, we can write the expansions up to the fourth order as follows:

$$
\rho_{n \pm 1}=\rho \pm \frac{1}{2} \rho_{x}+\frac{1}{8} \rho_{x x} \pm \frac{1}{48} \rho_{x x x}+\frac{1}{384} \rho_{x x x x} \pm \cdots,
$$

$$
\rho_{n \pm 2}=\rho \pm \rho_{x}+\frac{1}{2} \rho_{x x} \pm \frac{1}{6} \rho_{x x x}+\frac{1}{24} \rho_{x x x x} \pm \cdots,
$$

and the similar expansions up to the second order for the other lattice field $\eta_{n}(\tau)$. Note that we need only the secondorder expansions for the latter field because it belongs to the optical type. However, the relative displacement field $\rho_{n}(\tau)$ is of the acoustic type. It contains the negative dispersion of the longitudinal acoustic waves which appears due to the discreteness of the chain. Therefore we keep the fourth-order expansions of the field $\rho_{n}(\tau)$ in Eq. (9) whereas only its second-order expansions are required in Eq. (10). As for the other field $\eta_{n}(\tau)$, its second-order expansions in both Eqs. (9) and (10) are sufficient for the correct transition to the continuum limit. After some lengthy but straightforward "expansion", calculations we find that the resulting continuum version of Eqs. (9) and (10) can be represented through the generating functions (11) and (13) as follows:

$$
\begin{gathered}
P=P\left(z_{1}=\rho, z_{2}=\eta\right), \quad Q=Q\left(z_{1}=2 \rho, z_{2}=\eta_{x} / 2\right), \\
S=S\left(z_{1}=\rho, z_{2}=\eta\right), \quad T=T\left(z_{1}=2 \rho, z_{2}=\eta_{x} / 2\right) .
\end{gathered}
$$

Explicitly, we get

$$
P_{n+1}-2 P_{n}+P_{n-1}=\frac{1}{4}\left(1+\partial_{x}^{2} / 48\right) P_{x x},
$$




$$
\begin{gathered}
Q_{n+2}-Q_{n+1}-Q_{n}+Q_{n-1}=\frac{1}{2} Q_{x x}+\frac{1}{8} R, \\
S_{n-1}+2 S_{n}+S_{n+1}=4 S+\frac{1}{4} S_{x x}, \\
T_{n+1}-T_{n-1}+T_{n+2}-T_{n}=2 T_{x},
\end{gathered}
$$

where

$$
\begin{aligned}
R= & \frac{1}{3} \frac{\partial Q}{\partial \rho} \rho_{x x x x}+\frac{1 \partial^{2} Q}{4 \partial \rho^{2}}\left(3 \rho_{x x}^{2}+\frac{13}{3} \rho_{x} \rho_{x x x}\right) \\
& +\frac{11}{8} \frac{\partial^{3} Q}{\partial \rho^{3}} \rho_{x}^{2} \rho_{x x}+\frac{5}{24} \frac{\partial^{4} Q}{\partial \rho^{4}} \rho_{x}^{4} .
\end{aligned}
$$

Therefore, Eqs. (9) and (10) take the form

$$
\begin{gathered}
4 \rho_{\tau \tau}=(P+2 Q)_{x x}+\frac{1}{48} P_{x x x x}+\frac{1}{2} R, \\
\eta_{\tau \tau}+4 S+\frac{1}{4} S_{x x}-2 T_{x}=0 .
\end{gathered}
$$

The system of Eqs. (23) and (24) can further be simplified if we use the linearized form of the generating functions (13). In fact, in the next sections we will need their expansions including the next (nonlinear) terms. First, we expand the anharmonic potentials $U$ and $V$ up to their cubic terms. As a result, we obtain

$$
U(r)=\kappa_{1}\left(\frac{1}{2} r^{2}-\frac{\alpha}{3} r^{3}\right) \quad \text { and } V(q)=\kappa_{2}\left(\frac{1}{2} q^{2}-\frac{\beta}{3} q^{3}\right) .
$$

Next, we expand the generating functions (11) and (13) up to the second order. Using the definition (17), we find

$$
\begin{gathered}
P=\frac{\kappa_{1}}{4 b^{2}}\left(\rho-2 h \eta+A \rho^{2}+B \rho \eta+C \eta^{2}\right)+\cdots, \\
Q=\kappa_{2}\left(2 \rho-4 \beta \rho^{2}+\frac{1}{8} \eta_{x}^{2}\right)+\cdots \\
S=-\frac{h}{2 b^{2}} \kappa_{1}\left[\rho-2 h \eta+(A-2) \rho^{2}+\left(B+4 h-\frac{1}{h}\right) \rho \eta\right. \\
\left.+(C+2) \eta^{2}\right]+\cdots, \\
T=\kappa_{2} \rho \eta_{x}+\cdots
\end{gathered}
$$

where the constants $A, B$, and $C$ are given by

$$
\begin{gathered}
A=\frac{3 h^{2}}{b^{2}}-\frac{\alpha}{2 b}, \quad B=h\left(\frac{3}{b^{2}}-4+\frac{2 \alpha}{b}\right), \\
C=\frac{3}{4 b^{2}}-2-\frac{2 \alpha h^{2}}{b} .
\end{gathered}
$$

The final step in the procedure of deriving the equations of motion in the continuum limit is to linearize the highest derivative terms in both Eqs. (23) and (24). More exactly, in Eq. (23), we keep in the fourth-order derivative expressions only linear terms in $\rho$, i.e., those terms which are proportional to $\rho_{x x x x}$, and similarly, in the second-order derivative expressions we keep only linear terms in $\eta$. In Eq. (24), we linearize the second-order derivative terms in both $\rho$ and $\eta$. Therefore, according to the expansions (26)-(29), we can write the following approximations:

$$
P_{x x x x} \simeq \frac{\kappa_{1}}{4 b^{2}} \rho_{x x x x}, \quad R \simeq \frac{2}{3} \kappa_{2} \rho_{x x x x}
$$

in Eq. (23) and

$$
S_{x x} \simeq-\frac{h}{2 b^{2}} \kappa_{1}(\rho-2 h \eta)_{x x}, \quad T_{x} \simeq 0
$$

in Eq. (24). As a result, both Eqs. (23) and (24) are transformed to

$$
\begin{gathered}
4 \rho_{\tau \tau}-\left(P+2 Q+\frac{a_{1}}{3} \rho_{x x}\right)_{x x}=0, \\
\eta_{\tau \tau}-\frac{h}{8 b^{2}} \kappa_{1}(\rho-2 h \eta)_{x x}+4 S=0 .
\end{gathered}
$$

The constant $a_{1}$ in Eq. (33) takes into account the geometry of the zig-zag chain and it is given by

$$
a_{1}=\kappa_{1} / 64 b^{2}+\kappa_{2} .
$$

It should be noticed that Eq. (33) corresponds to the socalled ill-posed Boussinesq equation in the 1D case (see, e.g., Ref. [11] and other references therein). Similarly to the 1D case, an improved version of Eq. (33) can also be derived. In order to find it, we multiply both sides of Eq. (23) by the operator $\left(1-a_{2} \partial_{x}^{2} / 12\right)$ where the constant $a_{2}$ is to be determined from the requirement that all the fourth-order derivatives in the right-hand side (r.h.s.) of this equation have to be canceled. Then using the approximate expressions (31) as well as the relation

$$
Q_{x x x x} \simeq 2 \kappa_{2} \rho_{x x x x},
$$

which follows from the expansion (27), we obtain

$$
a_{2}=\frac{\kappa_{1} / 64 b^{2}+\kappa_{2}}{\kappa_{1} / 16 b^{2}+\kappa_{2}} .
$$

As a result, Eq. (23) is transformed to the equation

$$
4 \rho_{\tau \tau}-\left(P+2 Q+\frac{a_{2}}{3} \rho_{\tau \tau}\right)_{x x}=0
$$

which may be referred to as an improved version of Eq. (33). Notice that $0<a_{1}<\infty$ whereas $1 / 4<a_{2}<1$. Therefore, for the improved version (38), the constant $a_{2}$ appears to be more specified and less independent on the geometry of the chain and on the potentials $U$ and $V$ than for the ill-posed equation (33). 
Finally, Eqs. (33) or (38) can further be simplified if we take into account the form of the function $Q\left(z_{1}, z_{2}\right)$ in Eqs. (13) as well as the expansions (25) and (27). Then Eq. (38) becomes

$$
4 \rho_{\tau \tau}-\left[P+2 V^{\prime}(2 \rho)+\frac{a_{2}}{3} \rho_{\tau \tau}\right]_{x x}=0 .
$$

Thus, we have derived the pair of two coupled nonlinear field equations which describe the dynamics of the $2 \mathrm{D}$ zigzag chain. In what follows, we will study the improved version of the Boussinesq-like equation, i.e., Eqs. (34) and (39), where, according to Eqs. (11), (13), and (17), the functions $P$ and $S$ are defined by

$$
P=P(\rho, \eta)=\frac{1 / 2+\rho}{b+r} U^{\prime}(r) \text { and } S=S(\rho, \eta)=\frac{\eta-h}{b+r} U^{\prime}(r)
$$

with

$$
r=r(\rho, \eta)=\sqrt{\left(\frac{1}{2}+\rho\right)^{2}+(h-\eta)^{2}}-b
$$

It is interesting to consider different limiting cases of the continuum equations of motion (33) [or (39)] and (34). Consider the well-known 1D limit when only the longitudinal degrees of freedom with the nearest-neighbor interactions are kept, i.e., $\eta \rightarrow 0$ and Eq. (34) disappears. This limit can be realized when either (i) both the chains in the zig-zag backbone are decoupled, i.e., $U \rightarrow 0$, so that the first-neighbor interactions are ignored and only the second-neighbor ones are kept, or (ii) the zig-zag structure is shrinked, i.e., $h \rightarrow 0$ or $b \rightarrow 1 / 2$ and the second-neighbor interactions are omitted $(V \rightarrow 0)$.

In the first limiting case, $P \rightarrow 0, a_{1} \rightarrow \kappa_{2}=V^{\prime \prime}(0)$, and $a_{2} \rightarrow 1$. As a result, Eq. (39) as well as its ill-posed analog [see Eq. (33)] become

$$
\begin{aligned}
& 4 \rho_{\tau \tau}-2 V^{\prime}(2 \rho)_{x x}-\frac{V^{\prime \prime}(0)}{3} \rho_{x x x x}=0 \\
& \text { and } 4 \rho_{\tau \tau}-2 V^{\prime}(2 \rho)_{x x}-\frac{1}{3} \rho_{x x \tau \tau}=0 .
\end{aligned}
$$

Rescaling then the displacement field, $\rho \rightarrow \rho / 2$, we find that both Eqs. (42) are transformed to the well-known ill-posed and improved Boussinesq equations, respectively $[5,6,11]$.

In the other limiting case, $r \rightarrow \rho, P \rightarrow U^{\prime}(\rho), Q \rightarrow 0$, $a_{1} \rightarrow \kappa_{1} / 16=U^{\prime \prime}(0) / 16$, and $a_{2} \rightarrow 1 / 4$. As a result, Eqs. (33) and (39) are, respectively, reduced to

$$
\begin{aligned}
& 4 \rho_{\tau \tau}-U^{\prime}(\rho)_{x x}-\frac{U^{\prime \prime}(0)}{48} \rho_{x x x x}=0 \\
& \text { and } 4 \rho_{\tau \tau}-U^{\prime}(\rho)_{x x}-\frac{1}{12} \rho_{x x \tau \tau}=0 .
\end{aligned}
$$

Rescaling the lattice spacing, $x \rightarrow x / 2$, in both these equations, we find that they coincide with the ill-posed and improved Boussinesq equations, respectively.

\section{THE SMALL-AMPLITUDE LIMIT}

In this section we study the propagation of smallamplitude waves along the zig-zag backbone (see Fig. 1). The corresponding dispersion law can be derived from the linearized version of the basic equations of motion (9) and (10). Using the linearized form of the generating functions $P, Q, S$, and $T$, given by Eqs. (11) and (13), and by the definitions (14), we find

$$
\begin{gathered}
P_{n}=\frac{\kappa_{1}}{4 b^{2}}\left(\rho_{n}-2 h \eta_{n}\right), \quad Q_{n}=\kappa_{2}\left(\rho_{n-1}+\rho_{n}\right), \\
S_{n}=-\frac{h}{2 b^{2}} \kappa_{1}\left(\rho_{n}-2 h \eta_{n}\right), \quad T_{n}=0 .
\end{gathered}
$$

Substituting these relations into Eqs. (9) and (10), we get their linearized form

$$
\begin{gathered}
\frac{d^{2} \rho_{n}}{d \tau^{2}}=\frac{\kappa_{1}}{4 b^{2}}\left(\rho_{n+1}-2 \rho_{n}+\rho_{n-1}\right)+\kappa_{2}\left(\rho_{n+2}-2 \rho_{n}+\rho_{n-2}\right) \\
-\frac{h}{2 b^{2}} \kappa_{1}\left(\eta_{n+1}-2 \eta_{n}+\eta_{n-1}\right) \\
\frac{d^{2} \eta_{n}}{d \tau^{2}}=\frac{h}{2 b^{2}} \kappa_{1}\left(\rho_{n-1}+2 \rho_{n}+\rho_{n+1}\right) \\
-\frac{h^{2}}{b^{2}} \kappa_{1}\left(\eta_{n-1}+2 \eta_{n}+\eta_{n+1}\right)
\end{gathered}
$$

Next, substituting the plane waves

$$
\begin{gathered}
\rho_{n}=A_{1} e^{i(k n / 2-\Omega \tau)} \text { and } \eta_{n}=A_{2} e^{i(k n / 2-\Omega \tau)} \\
k \in[-2 \pi, 2 \pi],
\end{gathered}
$$

into Eqs. (45), we obtain the following linear dispersion equation:

$$
\begin{gathered}
\left(\Omega^{2}-\frac{\kappa_{1}}{b^{2}} \sin ^{2} \frac{k}{4}-4 \kappa_{2} \sin ^{2} \frac{k}{2}\right)\left(\Omega^{2}-\frac{4 h^{2}}{b^{2}} \kappa_{1} \cos ^{2} \frac{k}{4}\right) \\
-\left(\frac{h}{b^{2}} \kappa_{1} \sin \frac{k}{2}\right)^{2}=0 .
\end{gathered}
$$

In the decoupling limit, when $\kappa_{1} \rightarrow 0$, the dispersion equation (47) is reduced to the well-known linear dispersion law for a single linear chain:

$$
\Omega=2 \sqrt{\kappa_{2}}\left|\sin \frac{k}{2}\right|
$$

In the other particular case, when $\kappa_{2} \rightarrow 0$ and $h \rightarrow 0$ (and $b \rightarrow 1 / 2$ ), Eq. (47) is reduced to the other dispersion law

$$
\Omega=2 \sqrt{\kappa_{1}}\left|\sin \frac{k}{4}\right|
$$

which again corresponds to the linear chain if its lattice spacing is rescaled by the substitution $l \rightarrow l / 2$. 
In the general case, the dispersion law (47) describes the coupling of the two linear modes, i.e., the propagation of small-amplitude (i) longitudinal displacements $\rho_{n}(\tau)$ with the acoustical-like dispersion law,

$$
\Omega_{L}=2 \sqrt{\frac{\kappa_{1}}{4 b^{2}} \sin ^{2} \frac{k}{4}+\kappa_{2} \sin ^{2} \frac{k}{2}},
$$

and (ii) transverse displacements $\eta_{n}(\tau)$, with the optical-like dispersion relation

$$
\Omega_{T}=\frac{2 h}{b} \sqrt{\kappa_{1}} \cos \frac{k}{4} .
$$

The last term in Eq. (47) is due to the interaction between these modes. Since it is negative, a gap in the frequency spectrum (as shown in Fig. 2) appears. The explicit solution of the dispersion equation (47) gives two branches:

$$
\Omega_{a c, o p}^{2}=\kappa_{1}\left[1+\left(1-\frac{1}{2 b^{2}}\right) \cos \frac{k}{2}\right]+2 \kappa_{2} \sin ^{2} \frac{k}{2} \mp \sqrt{\left[\kappa_{1}\left(1-\frac{1}{2 b^{2}}+\cos \frac{k}{2}\right)-2 \kappa_{2} \sin ^{2} \frac{k}{2}\right]^{2}+\left(\frac{h}{b^{2}} \kappa_{1} \sin \frac{k}{2}\right)^{2}} .
$$

The upper sign ("“-”) in this equation corresponds to the low-frequency acoustical ( $\Omega_{a c}$-branch in Fig. 2) part of the frequency spectrum whereas the other sign ("+") gives the high-frequency optical ( $\Omega_{o p}$ branch in Fig. 2) part of this spectrum.

In the long-length-wave limit $(k \rightarrow 0)$ we have $\Omega_{a c}^{2} \rightarrow \kappa_{2} k^{2}$ and therefore the velocity of longitudinal sound along the chain is given by

$$
v_{0}=l \sqrt{K_{2} / M} \text { or } s_{0}=\sqrt{\kappa_{2}} \text {. }
$$

It is interesting to notice that this velocity coincides with the sound velocity for a single chain. The optical branch for small $k$ is described by the relation

$$
\Omega_{o p}^{2} \simeq \frac{4 h^{2}}{b^{2}} \kappa_{1}+\frac{1}{4}\left(\frac{1}{2 b^{2}}-1\right) \kappa_{1} k^{2}
$$
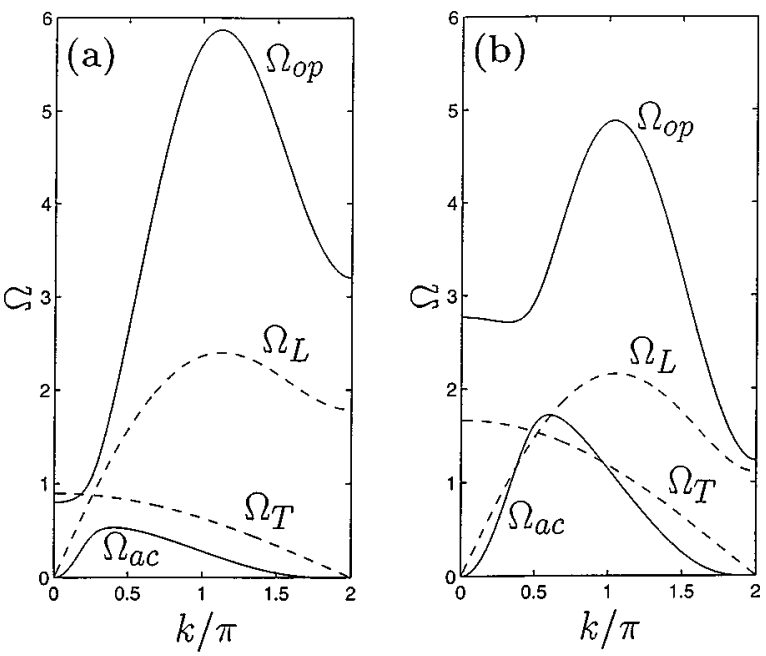

FIG. 2. Dependence of the dimensionless frequencies $\Omega_{a c, o p}$ (solid lines) and $\Omega_{L, T}$ (dashed lines) on the dimensionless wave number $k$ for the zig-zag chain with the parameters (a) $h=1 / 4$ (the case of positive dispersion of $\Omega_{o p}$ at small $k$ ) and (b) $h=3 / 4$ (the case of negative dispersion of $\Omega_{o p}$ at small $k$ ).
As follows from this relation, the long-length waves propagate with positive dispersion if $h<1 / 2$ [see Fig. 2(a)] and with negative dispersion if $h>1 / 2$ [see Fig. 2(b)].

\section{SOLITARY-WAVE SOLUTIONS}

In this section we will show that even in the case when both the intermolecular potentials $U$ and $V$ are harmonic, the zig-zag backbone admits solitary-wave solutions due to the effective nonlinearity which comes from the geometry of the configuration of the equilibrium positions of the chain molecules. It can clearly be seen from the expansions in Eqs. (7) when the next (anharmonic) terms are kept. This kind of nonlinearity may be referred to as "geometrical" anharmonicity [17] in order to distinguish it from the "intrinsic" anharmonicity of intermolecular interactions given, for instance, by the parameters $\alpha$ and $\beta$ in the expansions (25). As was mentioned above, we treat only the improved version, i.e., Eqs. (34) and (39). Substituting the expansions (25), (26), and (28), where the parameters $\alpha$ and $\beta$ are still kept, into these equations, we obtain

$$
\begin{gathered}
\rho_{\tau \tau}-\frac{a_{2}}{12} \rho_{x x \tau \tau}-\left[\left(\frac{\kappa_{1}}{16 b^{2}}+\kappa_{2}\right) \rho-\frac{h}{8 b^{2}} \kappa_{1} \eta\right. \\
\left.+\left(\frac{A}{16 b^{2}} \kappa_{1}-2 \beta \kappa_{2}\right) \rho^{2}+\frac{\kappa_{1}}{16 b^{2}}\left(B \rho \eta+C \eta^{2}\right)\right]_{x x}=0 \\
\eta_{\tau \tau}-\frac{h}{8 b^{2}} \kappa_{1}(\rho-2 h \eta)_{x x}-\frac{2 h}{b^{2}} \kappa_{1}\left[\rho-2 h \eta+(A-2) \rho^{2}\right. \\
\left.+\left(B+4 h-\frac{1}{h}\right) \rho \eta+(C+2) \eta^{2}\right]=0
\end{gathered}
$$

For the waves of a stationary profile moving with the dimensionless velocity $s=v / l \omega_{0}$ [see Eqs. (5)], Eqs. (55) and (56) are transformed to 


$$
\begin{aligned}
& \frac{4}{3} a_{2} b^{2} s^{2} \rho^{\prime \prime}+\left[\kappa_{1}+16 b^{2}\left(\kappa_{2}-s^{2}\right)\right] \rho-2 h \kappa_{1} \eta \\
& +\left(A \kappa_{1}-32 b^{2} \beta \kappa_{2}\right) \rho^{2}+\kappa_{1}\left(B \rho \eta+C \eta^{2}\right)=0, \\
& \rho^{\prime \prime}-2\left(h+\frac{4 b^{2}}{h \kappa_{1}} s^{2}\right) \eta^{\prime \prime}+16\left[\rho-2 h \eta+(A-2) \rho^{2}\right. \\
& \left.+\left(B+4 h-\frac{1}{h}\right) \rho \eta+(C+2) \eta^{2}\right]=0
\end{aligned}
$$

where the primes denote the differentiation of the fields $\rho(\xi)$ and $\eta(\xi)$ over the variable $\xi=x-s \tau$.

In order to treat the system of coupled nonlinear ordinary differential equations (57) and (58), we need to decouple them in some appropriate way. From the geometrical and physical points of view, it is reasonable to assume an approximate proportionality of both the fields $\rho(\xi)$ and $\eta(\xi)$, i.e., we can assume the following decoupling relation:

$$
\eta(\xi)=\chi \rho(\xi)
$$

where the parameter $\chi$ has to be determined and it should be positive. Indeed, if the longitudinal deformation $\rho(\xi)$ is a positive (negative) bell-shaped function, then the chain in the transverse direction should become thin (thicker).

Substituting the relation (59) into both Eqs. (57) and (58), we immediately find that they are reduced to the following two forms of the same Boussinesq-like equation:

$$
C_{0} \rho^{\prime \prime}+C_{1} \rho+C_{2} \rho^{2}=0 \text { and } D_{0} \rho^{\prime \prime}+D_{1} \rho+D_{2} \rho^{2}=0 .
$$

Here the two series of the constants are defined by

$$
\begin{gathered}
C_{0}=4 a_{2} b^{2} s^{2} / 3 \kappa_{1}, \\
C_{1}=1-2 h \chi+16 b^{2}\left(\kappa_{2}-s^{2}\right) / \kappa_{1}, \\
C_{2}=A+B \chi+C \chi^{2}-32 b^{2} \beta \kappa_{2} / \kappa_{1}
\end{gathered}
$$

and

$$
\begin{gathered}
D_{0}=\frac{1}{16}\left(2 h \chi-1+8 b^{2} s^{2} \chi / h \kappa_{1}\right), \\
D_{1}=2 h \chi-1, \\
D_{2}=2-A-\left(B+4 h-\frac{1}{h}\right) \chi-(C+2) \chi^{2} .
\end{gathered}
$$

Each of Eqs. (60) has the well-known soliton solution

$$
\rho(\xi)=\rho_{0} \cosh ^{-2}(\mu \xi),
$$

where the amplitude $\rho_{0}$ and the inverse half-width $\mu$ are given by the relations

$$
\rho_{0}=-\frac{3 C_{1}}{2 C_{2}}=-\frac{3 D_{1}}{2 D_{2}} \text { and } \mu^{2}=-\frac{C_{1}}{4 C_{0}}=-\frac{D_{1}}{4 D_{0}} .
$$

The compatibility of the two relations (64) results in the following equation with respect to the coefficient $\chi$ :

$$
\frac{s^{2}-\kappa_{2}+(2 h \chi-1) \kappa_{1} / 16 b^{2}}{4 a_{2} s^{2} / 3}=\frac{1-2 h \chi}{2 h \chi-1+8 b^{2} s^{2} \chi / h \kappa_{1}},
$$

where the velocity $s$ satisfies the equation

$$
s^{2}=\kappa_{2}+\frac{\kappa_{1}}{16 b^{2}}(1-2 h \chi)\left(1+\frac{C_{2}}{D_{2}}\right) \text {. }
$$

Since $\mu^{2}>0$, both sides of Eq. (65) must be positive. The positivity of the r.h.s. of this equation leads to the inequality $1-2 h \chi>0$, so that the coefficient $\chi$ must be in the interval

$$
0<\chi<1 / 2 h
$$

On the other hand, the positivity of the 1.h.s. of Eq. (65) implies that only supersonic $\left(s>s_{0}=\sqrt{\kappa_{2}}\right)$ velocities are appropriate solutions of Eqs. (65) and (66).

Finally, substituting the expression for $s^{2}$ given by Eq. (66) into Eq. (65), we find the following algebraic equation of the sixth order with respect to the coefficient $\chi$ :

$$
\begin{aligned}
\mathcal{P}(\chi) \equiv & (1-2 h \chi)\left[3 G_{1}\left(h \chi G_{1}+\kappa_{1} \chi G_{2}-2 h \kappa_{1} G_{2}\right)\right. \\
& \left.-8 a_{2} \kappa_{1} G_{2}\left(h G_{1}+\kappa_{1} G_{2}\right)\right] \\
& +16 b^{2} \kappa_{2} G_{2}\left(3 \chi G_{1}-8 a_{2} \kappa_{1} G_{2}\right)=0
\end{aligned}
$$

where $G_{1}=\kappa_{1} C_{2}$ and $G_{2}=h D_{2}$. Only those roots of Eq. (68), which satisfy the inequalities (67) and provide the inequality $s>\sqrt{\kappa_{2}}$, must be chosen.

Since the constant $\chi$ has to be always positive, it follows from Eq. (59) that $t w o$ types of soliton solutions can exist. If the amplitude of the longitudinal soliton component $\rho_{0}$ is found to be positive, then the amplitude of the transverse component $\eta_{0}$ is also positive. In this case, the soliton solution describes a localized longitudinal stretching which is accompanied by a localized slendering $\left(\eta_{0}>0\right)$. In fact, this transverse slendering occurs with the amplitude $\eta_{0}$ exceeding the thickness $h$.

In what follows, the two-component soliton solutions will be called accordingly to the type of longitudinal deformation. Therefore a solitary-wave solution, the longitudinal component of which is a positive function, will be referred to as a stretching soliton. On the other hand, according to Eq. (59), a localized longitudinal compression can coexist with a transverse thickening (bulge) of the chain. We call this type of localized deformation a compression soliton. Note that the latter type of solitons resembles the conventional lattice solitons in a 1D anharmonic chain.

It follows from Eqs. (30), (62), (64), (66), and (67) that even in the case when the intrinsic anharmonicity is absent, i.e., $\alpha=\beta=0$, the stretching solitary-wave solution exists. Indeed, in the particular case $\alpha=0$ the corresponding inequalities $\rho_{0}>0$ and $s>s_{0}$ imply

$$
D_{2}=\frac{1 / 2-h^{2}}{b^{2}}+\left(1-\frac{3 h^{2}}{b^{2}}\right) \frac{\chi}{h}-\frac{3}{4 b^{2}} \chi^{2}>0,
$$



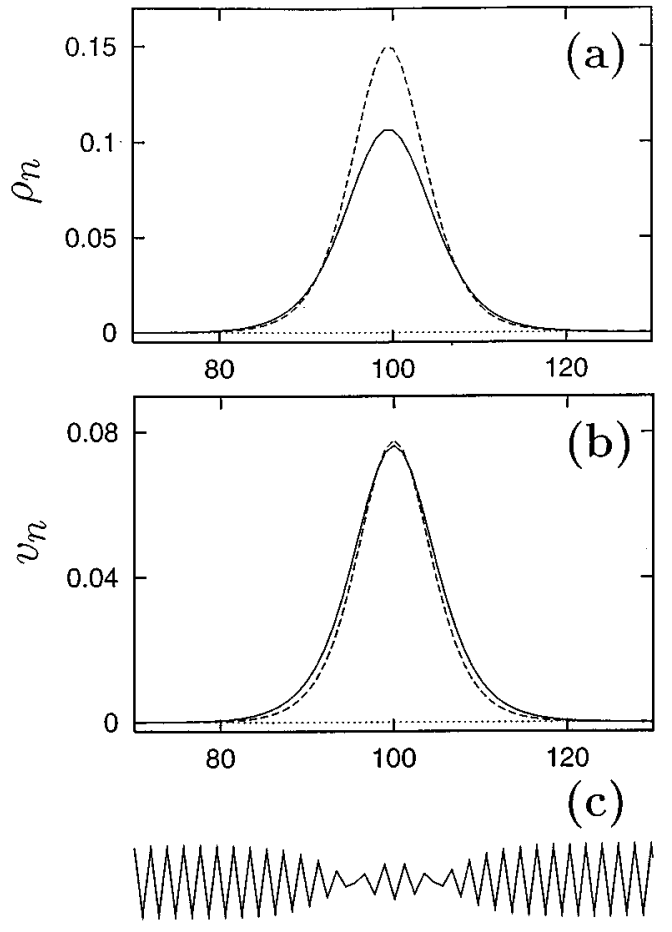

$n$

FIG. 3. The profiles of (a) longitudinal and (b) transverse displacements for the stretching soliton in the zig-zag chain with $h=0.1, \kappa_{1}=\kappa_{2}=1$, and $\alpha=\beta=0$, including (c) schematic representation of the chain deformation. The dashed lines show the soliton profiles obtained as a result of solving the sixth-order algebraic equation (68). The solid lines represent the result of solving the minimization problem for the function (80) at the velocity $s=s_{2}=1.10259<s_{1}$.

$$
C_{2}+D_{2}=2\left(1-\chi^{2}\right)+\left(1-4 h^{2}\right) \frac{\chi}{h}-32 b^{2} \beta \frac{\kappa_{2}}{\kappa_{1}}>0 .
$$

For sufficiently small $h$ both these inequalities can obviously be fulfilled even if we put $\beta=0$ in Eq. (70). Thus, for the zig-zag chain with the parameter values $h=0.1$, $\kappa_{1}=\kappa_{2}=1$, and $\alpha=\beta=0$, we have found two roots in the interval (67). However, only one of them corresponds to a supersonic velocity $s=s_{1}$. This root is $\chi=\chi_{1}=1.32$ and the corresponding value for the soliton velocity $s$ is calculated from Eqs. (66), (69), and (70): $s=s_{1}=1.11$. Next, $\rho_{0}=0.14$ and $\mu=0.33$, and since the inequality (69) is fulfilled, this solution describes a stretching soliton. Its longitudinal and transverse profiles are depicted, according to Eqs. (59) and (63), in Fig. 3 [the dashed lines in (a) and (b)]. Finally, as was mentioned above, the transverse amplitude of the stretching soliton $\eta_{0}$ always exceeds the thickness $h$ and, as a result of such "overslendering," the transverse deformation [see Fig. 3(c)] has two nodes [where $\eta(\xi)=h]$ and a bulge between them [where $\eta(\xi)>h$ ].

For the existence of the second type of solitary-wave solutions, compression solitons with a localized transverse thickening, the inequalities

$$
D_{2}<0 \text { and } C_{2}+D_{2}<0
$$

have to be satisfied. As follows from the expressions for $D_{2}$ and $C_{2}+D_{2}$ given in Eqs. (69) and (70), for sufficiently large anharmonicity $\beta$ and sufficiently large thickness $h$ the inequalities (71) can be fulfilled. Therefore, for each chain there exists a certain threshold of longitudinal anharmonicity, above which the compression solitons can exist. On the other hand, for sufficiently small $h$ (thin chains) the solitons cannot exist and this phenomenon can be explained as their transverse instability, as was pointed out in Ref. [7] under numerical simulations. Note that in the 1D theory of nonlinear lattices the solitons exist at any positive anharmonicity and with any supersonic velocity (i.e., there are no gaps in the soliton velocity spectrum).

Finally, it should be noticed that the existence of soliton solutions, with some fixed values of their velocity, within the ansatz (59) does not yet imply either their dynamical stability at these velocities or the existence of a discrete velocity spectrum. The appearance of the fixed soliton velocities is merely a result of the decoupling procedure of Eqs. (57) and (58) by using the constraint (59). In order to find exactly all soliton solutions, some specific numerical techniques will be developed in the next section.

\section{A NUMERICAL METHOD}

In this section we develop a numerical minimization scheme for seeking solitary-wave solutions of a stationary profile for the basic equations of motion (9) and (10). When these solutions have been found, they can be chosen as initial conditions for numerical simulations of these equations. Then a final profile of the lattice fields $\rho_{n}(\tau)$ and $\eta_{n}(\tau)$ obtained under the simulations at sufficiently large times $\tau$ allows us to conclude whether or not the initial condition found by the minimization procedure is a correct stable solution of Eqs. (9) and (10). The main point in such a numerical approach is an appropriate choice of a discrete functional for minimization. As was shown previously (see, e.g., Refs. $[18,19]$ ), such a functional can be constructed from the corresponding Lagrangian of the system. However, the zig-zag backbone studied in this paper appears to be a rather complicated system and therefore the numerical scheme developed previously in Refs. $[18,19]$ cannot be applied directly for seeking solitary-wave solutions in this system.

The (dimensionless) Lagrangian, which corresponds to the Hamiltonian (1), has the form

$$
\begin{aligned}
\mathcal{L} & =\mathcal{L}\left\{\frac{d u_{n}}{d \tau}, u_{n} ; \frac{d v_{n}}{d \tau}, v_{n}\right\} \\
& =\sum_{n}\left\{\frac{1}{2}\left[\left(\frac{d u_{n}}{d \tau}\right)^{2}+\left(\frac{d v_{n}}{d \tau}\right)^{2}\right]-U\left(r_{n}\right)-V\left(q_{n}\right)\right\} .
\end{aligned}
$$

The main idea in our numerical scheme is to replace the time derivatives $d u_{n} / d \tau$ and $d v_{n} / d \tau$ in the Lagrangian (72) by appropriate spatial differences of the lattice fields $\rho_{n}(\tau)$ and $\eta_{n}(\tau)$. Such a replacing procedure can be applied to those lattice functions which (i) are sufficiently smooth from site to site and (ii) have a stationary profile moving with velocity 
$s$. According to the previuos studies (see Refs. $[18,19]$ ), the simplest choice of such an approximation would be as follows:

$$
\frac{d u_{n}}{d \tau} \simeq-s u^{\prime}(n-s \tau) \simeq-2 s\left(u_{n+1}-u_{n}\right)=-2 s \rho_{n}
$$

and

$$
\frac{d v_{n}}{d \tau} \simeq-s v^{\prime}(n-s \tau) \simeq-2 s\left(v_{n+1}-v_{n}\right) .
$$

In this case, we can obtain the finite-dimensional Lagrangian function $\mathcal{L}=\mathcal{L}\left\{\rho_{n}, v_{n}\right\}$ which can be studied by the steepest descent method. However, for our zig-zag model the approximation defined by Eqs. (73) and (74) is too crude because it does not contain the lattice dispersion of the longitudinal waves.

In order to improve the numerical scheme, the second time derivative in Eq. (9) has to contain also the fourth-order spatial derivative with an appropriate coefficient chosen in such a way that the continuum limit (up to the fourth-order expansions) of the resulting discrete equation would coincide completely with the continuum version (33) or (38). Therefore, we write the following improved discretization as compared with Eq. (73):

$$
\begin{aligned}
\frac{d^{2} \rho_{n}}{d \tau^{2}} \simeq & 4 s^{2}\left[\rho_{n+1}-2 \rho_{n}+\rho_{n-1}\right. \\
& \left.-c\left(\rho_{n+2}-4 \rho_{n+1}+6 \rho_{n}-4 \rho_{n-1}+\rho_{n-2}\right)\right],
\end{aligned}
$$

where the constant $c$ is to be determined. The last group of terms in Eq. (75) is the fourth-order difference derivative which has to cancel the corresponding fourth-order continuum derivative appearing from the first group in this equation. As a result, we find $c=1 / 12$ and then in the continuum limit (up to the fourth order) Eq. (75) is transformed to $d^{2} \rho_{n} / d \tau^{2} \simeq s^{2} \rho^{\prime \prime}$. Consequently, the set of Eqs. (9) and (10) can be replaced by the following difference equations:

$$
\begin{aligned}
& 4 s^{2}\left[\rho_{n+1}\right.-2 \rho_{n}+\rho_{n-1}-\frac{1}{12} \\
& \quad \times\left.\left(\rho_{n+2}-4 \rho_{n+1}+6 \rho_{n}-4 \rho_{n-1}+\rho_{n-2}\right)\right] \\
& \quad=P_{n+1}-2 P_{n}+P_{n-1}+Q_{n+2}-Q_{n+1}-Q_{n}+Q_{n-1},
\end{aligned}
$$

$$
\begin{aligned}
4 s^{2}\left(\eta_{n+1}-2 \eta_{n}+\eta_{n-1}\right)= & -\left(S_{n-1}+2 S_{n}+S_{n+1}\right)+T_{n+1} \\
& -T_{n-1}+T_{n+2}-T_{n} .
\end{aligned}
$$

The left-hand sides of these equations represent the discretized second-order time derivatives in Eqs. (9) and (10). Notice that the last equation could also be discretized in the same way as Eq. (76) in order to take into account the lattice dispersion of transverse vibrations. However, as was shown before, this dispersion is not "responsible" for the solitons and therefore it is not necessary to use this more complicated discretized version. In fact, we have checked the results of both these procedures and did not find any significant difference while the presence of the fourth-order difference term in the 1.h.s. of Eq. (76) is crucial.

The difference equations (76) and (77) can be twice "integrated" and transformed to

$$
\begin{gathered}
4 s^{2}\left[\rho_{n}-\frac{1}{12}\left(\rho_{n+1}-2 \rho_{n}+\rho_{n-1}\right)\right]-P_{n}-Q_{n}-Q_{n+1}=0, \\
4 s^{2}\left(v_{n+1}-2 v_{n}+v_{n-1}\right)+S_{n-1}+S_{n}+T_{n-1}-T_{n+1} .
\end{gathered}
$$

These equations correspond to the Lagrangian function

$$
\begin{aligned}
\mathcal{L}= & \mathcal{L}\left\{\rho_{n}, v_{n}\right\} \\
= & \sum_{n}\left\{2 s^{2}\left[\rho_{n}^{2}+\frac{1}{12}\left(\rho_{n+1}-\rho_{n}\right)^{2}+\left(v_{n+1}-v_{n}\right)^{2}\right]\right. \\
& \left.-U\left(r_{n}\right)-V\left(q_{n}\right)\right\} .
\end{aligned}
$$

The extrenum conditions $\partial \mathcal{L} / \partial \rho_{n}=0$ and $\partial \mathcal{L} / \partial v_{n}=0$ give immediately the system of the difference equations (78).

However, the extrenum points of the Lagrangian function (79), which correspond to soliton solutions, are not necessary to be minima or maxima. They might be saddle points as well. For instance, for the 1D Boussinesq equation, there are neither minimum nor maximum points at the corresponding Lagrangian surface. In this case, the extrenum points which correspond to the supersonic soliton solutions are only of the saddle-type, and therefore it is impossible to find any supersonic solution by minimization of the function (79). However, in this case we can make a "reflection" of the Lagrangian surface in such a way that saddle points are transformed into minimum points at this "deformed" surface. The most direct and simple way is to construct the functional

$$
\mathcal{F}=\mathcal{F}\left\{\rho_{n}, v_{n}\right\}=\frac{1}{2} \sum_{n}\left[\left(\frac{\partial \mathcal{L}}{\partial \rho_{n}}\right)^{2}+\left(\frac{\partial \mathcal{L}}{\partial v_{n}}\right)^{2}\right]
$$

and then to study it for minima. Those minima which correspond to bell-shaped profiles are chosen as appropriate solitary-wave solutions of our problem. Other minima describing bell-shaped configurations accompanied by any ripples, etc., are to be excluded from the further consideration.

The analytical solution found in the preceding section can be chosen as a starting point for the descent. The minimization process is over when the discrete functional (80) reaches its minimum. It should be noticed that instead of the function (79) we could use the corresponding discretized Lagrangian $\mathcal{L}\left\{\rho_{n}, \eta_{n}\right\}$. However, in this case we would lose some dispersion terms in a final discretized version of the Lagrangian. More exactly, instead of the sum $S_{n-1}+2 S_{n}+S_{n+1}$ in Eq. (77) [or Eq. (10)], we would obtain only the term $4 S_{n}$.

\section{NUMERICAL RESULTS}

We have numerically studied the zig-zag system in both cases: (i) without any intrinsic anharmonicity, when the molecules are assumed to be coupled harmonically $(\alpha=\beta=0)$, and (ii) with this type of anharmonicity, when the parameter 

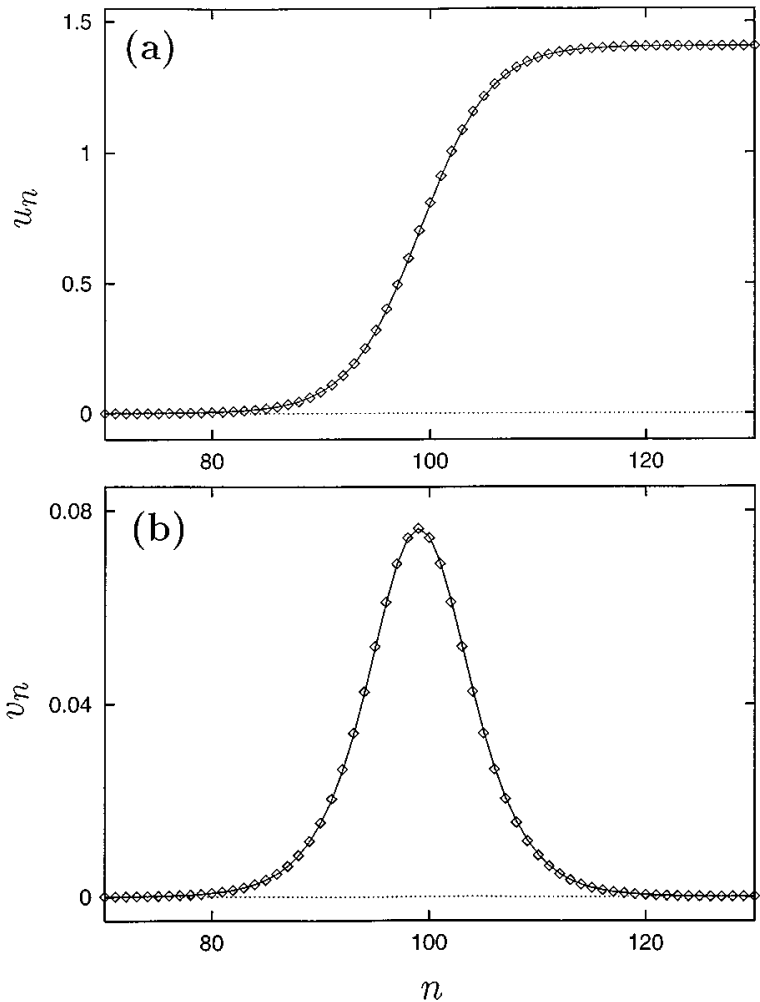

FIG. 4. The two-component soliton profiles given by the longitudinal $\left(u_{n}\right)$ and transverse $\left(v_{n}\right)$ displacements. The initial profile (at $\tau=0$ ) has been found by the minimization of the function (80) and it is represented by dots. The final profile (solid lines) is a result of evolution of this profile [according to the equations of motion (4)] when the soliton has passed 100000 chain sites (at time $\tau=45348.8$ ).

$\beta$ is positive. In the former case, we have chosen the zig-zag geometry with $h=0.1$ by taking $\kappa_{1}=\kappa_{2}=1$. The numerical minimization of the functional (79) has shown that the system has the soliton solution only at one value of the velocity $s=s_{2}=1.10259$. The minima at other values of the velocity $s$ do not provide pure bell-shaped soliton profiles; as a rule, they give profiles with ripples. The accuracy of the solution was reached up to $10^{-10}$. The results of the minimization are plotted in Fig. 3 by the solid lines.

The dynamics of the soliton solutions has been studied numerically in the zig-zag chain consisting of $N=300 \mathrm{~mol}-$ ecules, with the free ends. The accuracy of integration of Eqs. (4), rewritten in terms of the dimensionless lattice fields $u_{n}(\tau)$ and $v_{n}(\tau)$, was estimated through the conservation of the total energy given by the Hamiltonian (1). Initially, the soliton was situated at the site $n=100$. After the passage of 100 sites, the fields $u_{n}(\tau)$ and $v_{n}(\tau)$ were replaced by $u_{n-100}(\tau)$ and $v_{n-100}(\tau)$, respectively. The similar substitution was also accomplished with the time derivatives of these fields. This procedure allows us to cut off nonsoliton contributions appearing due to emission of small-amplitude waves.

The numerical simulation of the equations of motion has proved the stability of propagation of the stretching soliton. Thus, it was shown that starting with the initial velocity $s=s_{2}$, the soliton propagation with the final velocity $s=\bar{s}=1.10257$ was extremely stable as illustrated by Fig. 4 . Thus, after the soliton passed 100000 lattice sites, during the

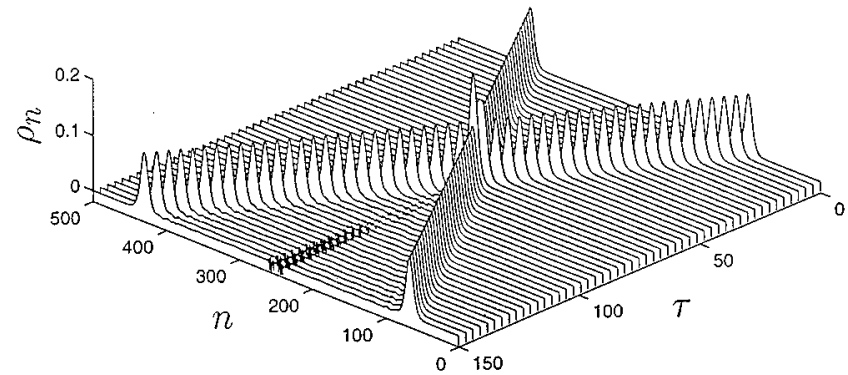

(a)

(b)

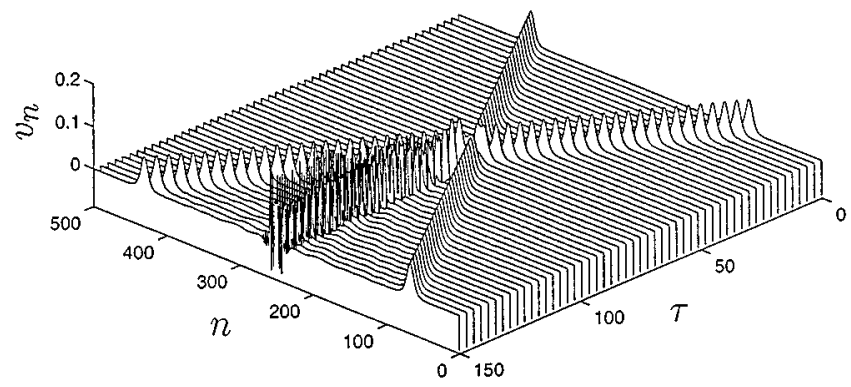

FIG. 5. Inelastic interaction of the stretching solitons under their head-in collision with velocity $s=1.10257\left(h=0.1, \kappa_{1}=\kappa_{2}=1\right.$, and $\alpha=\beta=0$ ).

time $\tau=45348.8$, its velocity $s=\bar{s}$ and two-component profile plotted in Fig. 4 by the solid lines were not changed. As illustrated in this figure, the initial profile found by the minimization scheme completely coincides with the final profile obtained as a result of the numerical simulation of the equations of motion. For all other velocities, either the soliton stabilizes its motion with the velocity $s=\bar{s}$ leaving smallamplitude ripples or it decays into a subsonic wave packet. Thus, if we take the analytical solution, obtained in the continuum limit and given by Eqs. (63) and (64), with $h=0.1$, $\kappa_{1}=\kappa_{2}=1$, and $\alpha=\beta=0$, as an initial profile, then the soliton propagation, which starts with any velocity $s>\bar{s}$, is accompanied by emission of small-amplitude waves and its velocity decreases approaching the value $s=\bar{s}$. The intensity of emission of small-amplitude waves also decreases and the soliton motion is stabilized. At any velocity $s<\bar{s}$, the soliton motion is also accompanied by emission of small-amplitude waves which results in gradually breaking and finally destroying the soliton.

The effects of interaction of the stretching solitons have also been investigated by numerical simulations. To this end, we have simulated their head-in collision. This collision leads to the appearance of transverse vibrations of the chain molecules. The results of these simulations are illustrated in Fig. 5. They show that the stretching solitons are sensitive with respect to their mutual collisions resulting in the emission of transverse oscillations (optical phonons).

Consider now the second type of the solitons which describe a localized longitudinal compression of the zig-zag chain. As was pointed out in Sec. V, these solitons can exist 

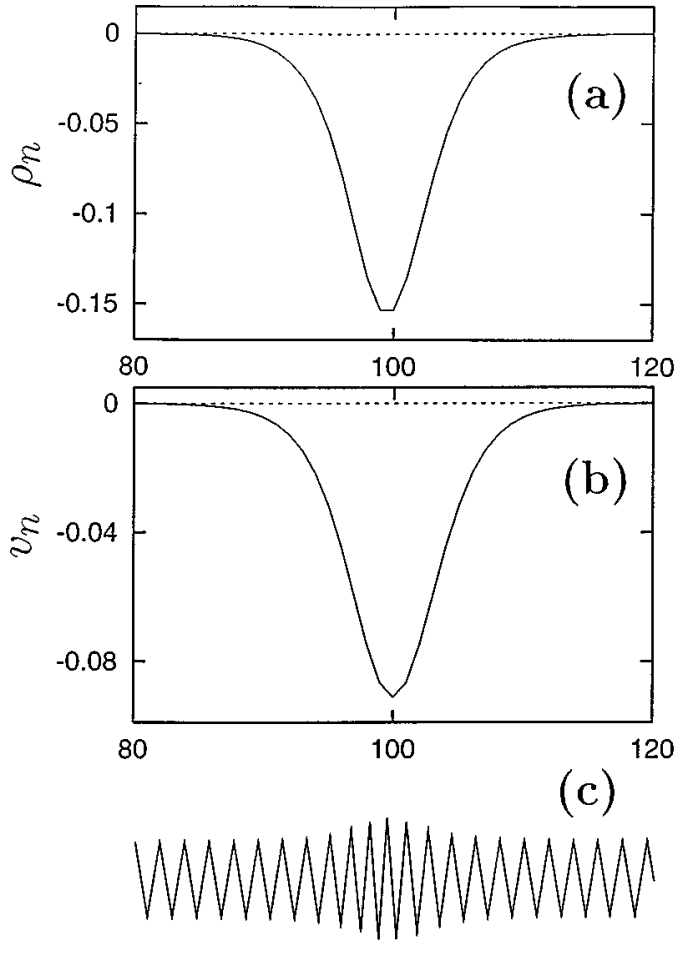

$n$

FIG. 6. The bell-shaped profiles of the compression soliton which are represented by the lattice fields of (a) longitudinal relative displacements $\rho_{n}$ and (b) transverse displacements $v_{n}$ ( $h=1 / 2 \sqrt{3}, \kappa_{1}=1$ and $\kappa_{2}=0.1, \alpha=0$, and $\beta=0.1$ ). These profiles describe (c) a longitudinal compression and a transverse thickening. The soliton has the velocity $s / s_{0}=1.1$, the energy $\mathcal{E}=0.06$, and the diameter $\mathcal{D}=7.75$.

only in the case if the longitudinal anharmonicity (with the parameter $\beta$ ) is sufficiently strong. Take the most typical situation for molecular chains when the ratio $\kappa_{1} / \kappa_{2}$ is of one order. For instance, in alpha-helix protein the constant $\kappa_{1}$ corresponds to hard deformations of the valence bonds while the other constant $\kappa_{2}$ determines soft vibrations of the hydrogen bonds. It is reasonable to consider the anharmonicity only in the soft bonds because they can have large amplitudes of deformation. Therefore we take $\alpha=0$ and $\beta>0$. Let $\kappa_{1}=1, \kappa_{2}=0.1$, and $h=1 / 2 \sqrt{3}$ (corresponding to the zig-zag angle $120^{\circ}$ ). The following three values of the parameter $\beta$ have been chosen: $\beta=0.1, \beta=0.01$, and $\beta=0.001$. The numerical solution of the minimization of the function (80) has shown that the compression solitons can exist only for sufficiently strong anharmonicity in the zig-zag chain. The typical two-component profile of the compression soliton is presented in Fig. 6. As demonstrated here, in the localized region of the soliton, a longitudinal compression and a transverse thickening take place.

Contrary to the 1D anharmonic chain, the velocity spectrum of the compression soliton in the zig-zag chain consists of a narrow band in the supersonic region which turns out to be bounded above. Thus, we have obtained the following velocity bands: $1<s / s_{0}<1.16$ if $\beta=0.1$ and $1<s / s_{0}<1.03$ if $\beta=0.01$. The dependence of the energy $\mathcal{E}$, the diameter $\mathcal{D}$, as well as the amplitudes of the longitudinal $\left(\mathcal{A}_{L}\right)$ and
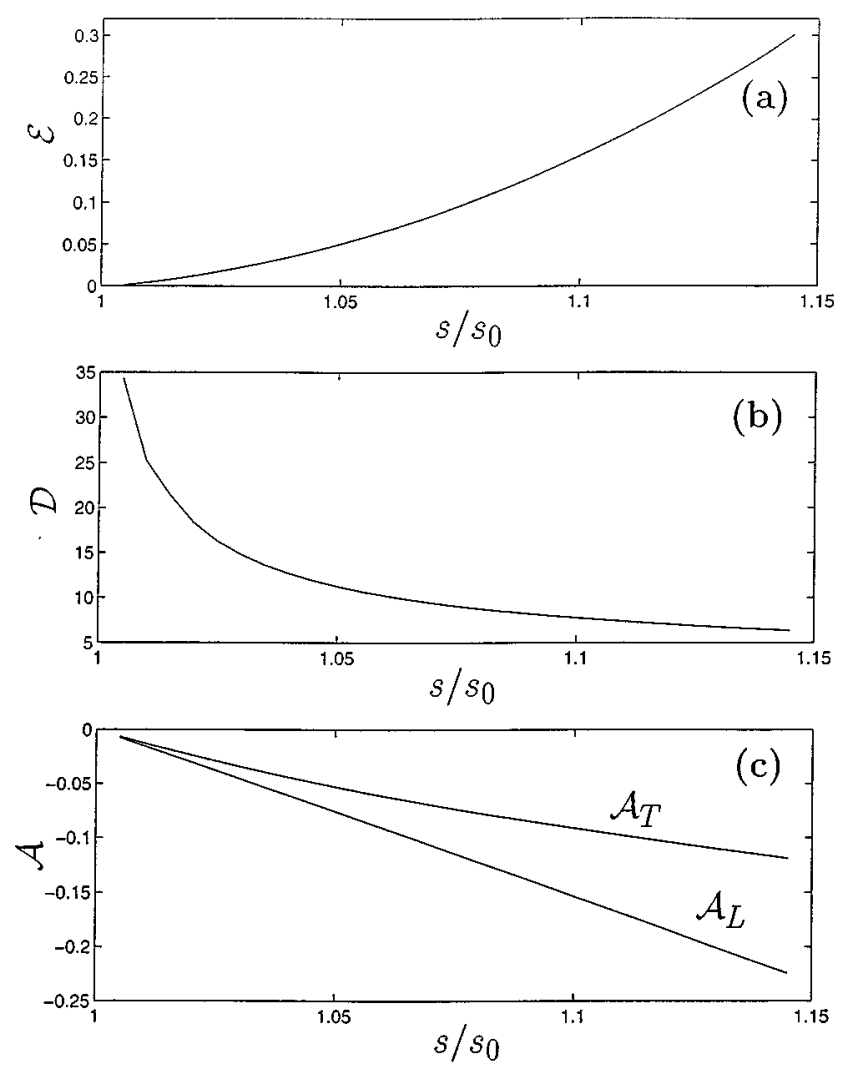

FIG. 7. Dependence of the energy $\mathcal{E}$, the diameter $\mathcal{D}$, and the amplitudes $\mathcal{A}_{\mathcal{L}}$ and $\mathcal{A}_{\mathcal{T}}$ of the compression soliton on its velocity at $\beta=0.1$.

transverse $\left(\mathcal{A}_{T}\right)$ soliton components on the velocity $s$ are shown in Fig. 7 (for $\beta=0.1$ ) and in Fig. 8 (for $\beta=0.01$ ).

The numerical simulations have also shown that the compression solitons are dynamically stable for the anharmonicity parameter $\beta=0.1$. Thus, at the initial velocity $s / s_{0}=1.1$ the soliton propagates along 100000 chain cells during the period $\tau=143826.8$. The soliton was moving with the velocity $s / s_{0}=1.0993$ retaining its profile as illustrated by Fig. 9. The solitons of this type have been proved to interact elastically (see Fig. 10).

The compression solitons in the chain with a weak anharmonicity (for instance, with $\beta=0.01$ ) propagate as stable objects only for velocities from the interval $1<s / s_{0}<1.015$. However, their interaction appears to be inelastic; after the collision the emission of transverse smallamplitude waves (optical phonons) occurs (see Fig. 11).

Thus, the numerical studies have proved that in an anharmonic zig-zag chain the existence of the compression solitons depends on the magnitude of the anharmonicity parameter $\beta$. For each zig-zag chain there exists a threshold value of this parameter, above which the compression solitons do exist. The solitons of this type have always a finite band of supersonic velocities. This band is enlarged with increasing the parameter $\beta$. The solitons lose their dynamical stability at the upper edge of the band. Below this edge, with decreasing velocity $s$, the compression solitons become dynamically stable objects, interacting as elastic particles. 

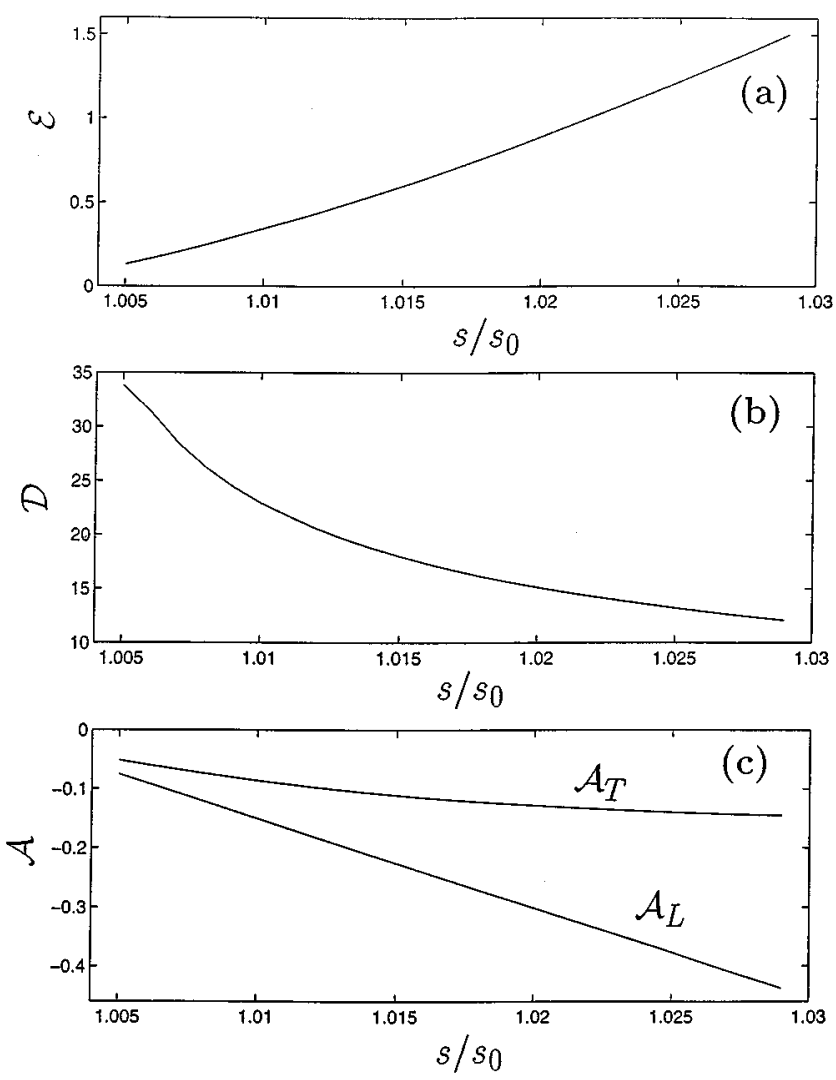

FIG. 8. Dependence of the energy $\mathcal{E}$, the diameter $\mathcal{D}$, and the amplitudes $\mathcal{A}_{\mathcal{L}}$ and $\mathcal{A}_{\mathcal{T}}$ of the compression soliton on its velocity at $\beta=0.01$.

\section{DISCUSSION AND CONCLUSIONS}

It was an attractive point of view to study some dynamical processes in biopolymers on the basis of the 1D nonlinear lattice models with nearest-neighbor interatomic interactions which admit propagation of extremely stable supersonic pulses of longitudinal lattice compression. Thus, Yomosa essentially applied the Fermi-Pasta-Ulam model for studies of energy transport in protein [20]. However, since any biopolymer should be considered as a 3D object, its dynamics has to include also the transverse degrees of freedom of the chain molecules, as was suggested, for instance, in Refs. [7-10].

Once (i) the transverse motions of the chain molecules are allowed and (ii) all the intermolecular forces in the system are assumed to have spherical symmetry, then some additional intermolecular forces, including the next or more remote neighbors and forming a secondary structure, have to be taken into account in order to stabilize some regular spatial configuration of the chain. In the simplest case, such a configuration has naturally the form of a so-called 310 -helix [7]. From the point of view of analytical studies, we have simplified the helical structure as much as possible by keeping the main features of the secondary structure. More precisely, our simplification has been done by (i) reducing one transverse coordinate and (ii) taking into account only the second-neighbor intermolecular interactions. Such an oversimplified "planar helix" is nothing more than a 2D zig-zag chain in which the first- and second-neighbor molecules are coupled by intermolecular interactions. All the molecules of
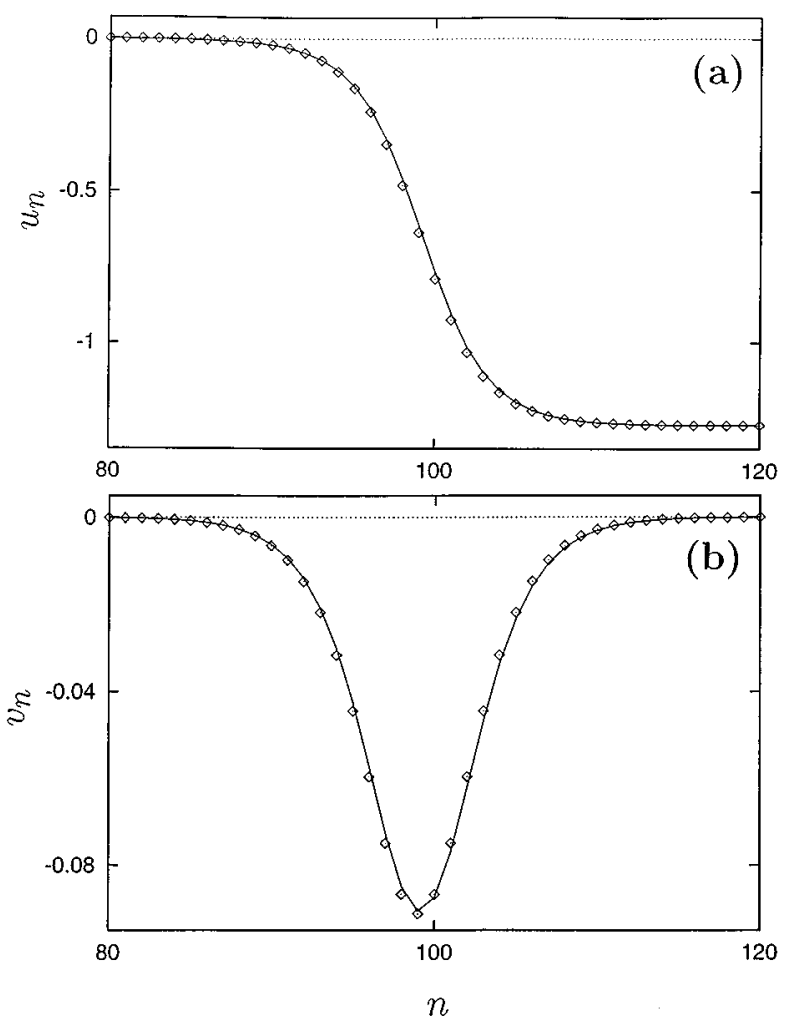

FIG. 9. The two-component profiles of the compression soliton represented by the displacement fields $u_{n}$ and $v_{n}$. The initial profile (shown by dots) at $\tau=0$ has been found by the minimization procedure while the final profile (solid lines) has been obtained as a result of time evolution of this initial profile after the passage of 100000 chain sites (at $\tau=143$ 826.8). The soliton velocity is given by $s / s_{0}=1.1$; the other parameters are $h=1 / 2 \sqrt{3}, \kappa_{1}=1$ and $\kappa_{2}=0.1, \alpha=0$, and $\beta=0.1$.

such a zig-zag chain were supposed to have two degrees of freedom: longitudinal $\left(x_{n}\right)$ and transverse $\left(y_{n}\right)$. The essence of this model is that the basic equations of motion (9) and (10) can be treated analytically in the continuum limit. On the other hand, from the point of view of the soliton stability studies some numerical techniques appeared to be also useful. Thus, the numerical minimization scheme which allows us to find pure soliton solutions in nonlinear models of big complexity has been suggested and developed in this paper. The analytical results have been used as starting points under the minimization procedure.

We do not expect any significant qualitative difference (in the dynamical behavior) between the 3D helix and its oversimplified zig-zag version. However, under the "transition", from the $1 \mathrm{D}$ theory to the $2 \mathrm{D}$ case, some drastic changes in the soliton dynamics appear. Thus, compared to the 1D chain model where the compression solitons can propagate with any supersonic velocity, the corresponding two-component solitons (longitudinal compression and transverse thickening, see Fig. 6) in the zig-zag chain actually have a narrow band of supersonic velocities. The existence of the upper bound in the velocity spectrum follows from the geometry of the system. Furthermore, the solitons of this type were proved to collide elastically, as shown by Fig. 10, if their velocities are found at the lower edge of the velocity band. On the contrary, if they propagate with velocities at the upper edge, 
(a)

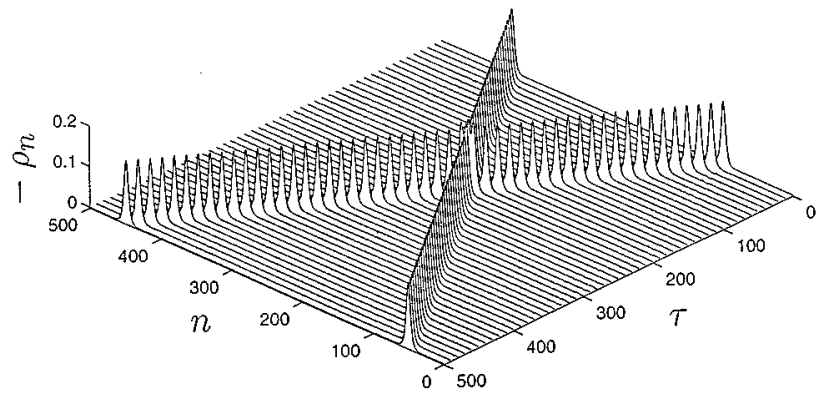

(b)

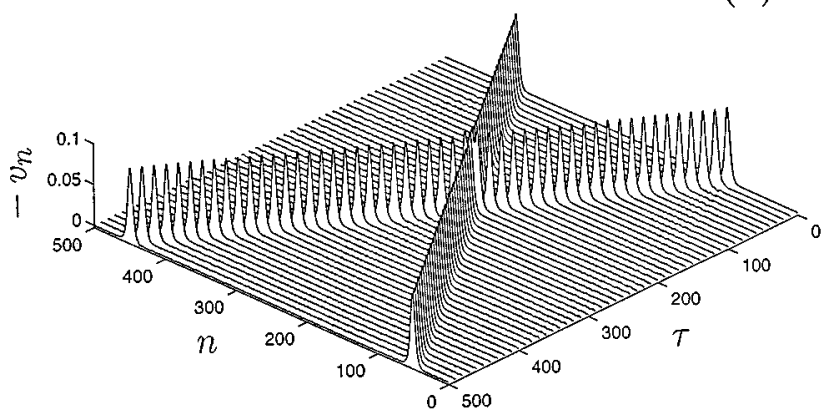

FIG. 10. Elastic head-in collision of two compression solitons with velocity $s / s_{0}=1.1\left(h=1 / 2 \sqrt{3}, \kappa_{1}=1\right.$ and $\kappa_{2}=0.1, \alpha=0$, and $\beta=0.1$.

their collision happens to be inelastic (see Fig. 11).

The next important observation in our studies is that the stability properties of the above-mentioned solitons essentially depend on both (i) the zig-zag chain geometry defined by its thickness $h$ and (ii) the magnitude of the intrinsic anharmonicity of the second-neighbor intermolecular forces (i.e., the parameter $\beta$ ). As was shown analytically [see Eqs. (69) and (70)], the solitons with longitudinal compression cannot exist in the zig-zag chains with (i) small thickness $h$ or (ii) week anharmonicity $\beta$, while in a $1 \mathrm{D}$ chain they exist at any value of the intermolecular anharmonicity. The absence of the soliton solutions in this region of the parameters $h$ and $\beta$ is due to the transverse instabilities of the soliton motion which have previously been discovered in Refs. $[10,21]$.

However, instead of the solitons with a localized longitudinal compression, a stable soliton solution which corresponds to longitudinal stretching and transverse slendering (illustrated by Figs. 3 and 4) has been found analytically and numerically by using minimization techniques. As was proved in Sec. V, this solution appears due to the geometrical anharmonicity; it exists for thin chains with weak intrinsic anharmonicities including also the case with harmonic intermolecular forces $(\alpha=\beta=0)$. However, it should be noticed that the soliton amplitude of the transverse displacements, which are directed inside the zig-zag backbone, exceeds its thickness $h$. As a result of such transverse "overslendering,", the chain deformation takes the form which is schematically shown in Fig. 3(c). The transverse component of this soliton has two nodes with a bulge be- (a)

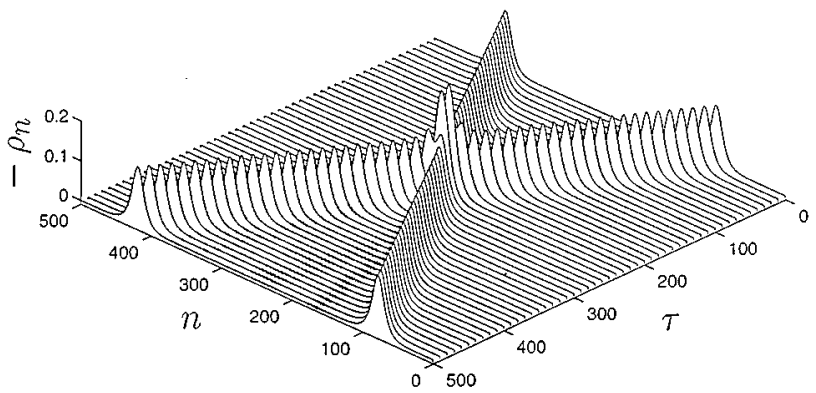

(b)

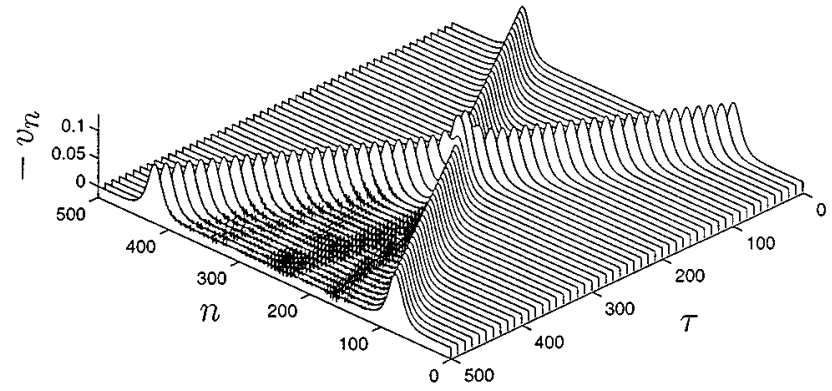

FIG. 11. Inelastic head-in collision of two compression solitons with velocity $s / s_{0}=1.01\left(h=1 / 2 \sqrt{3}, \kappa_{1}=1\right.$ and $\kappa_{2}=0.1, \alpha=0$, and $\beta=0.01$ ).

tween them where the "overslendering', occurs. The second interesting feature of this soliton is that there exists only one fixed velocity at which its stable propagation happens. This value of the soliton velocity has been found in this paper both analytically and numerically.

The existence of a single value of the soliton velocity can be explained from the physical point of view as follows. Roughly speaking, the soliton propagation along the zig-zag backbone can be considered as some superposition of two motions, one of which occurs only along the valence bonds while the second one happens along the hydrogen bonds. Each of these propagation processes has its own nonlinear dispersion law, i.e., some dependence of the amplitude of deformation on the velocity. On the other hand, both the amplitudes of the chain deformation are clearly related because of the zig-zag geometry. Therefore their relation imposes a constraint on the soliton velocity $s$. Both the amplitudes become self-consistent at some value of the velocity $s=\bar{s} \simeq s_{1} \simeq s_{2}$ which has been found in this paper. Of course, this value essentially depends on the geometrical structure and its exact value was found numerically.

It should be emphasized that in some more simple systems, the existence of a discrete velocity spectrum can be observed and explained much more easily. For instance, let us consider the motion of the lattice soliton in the on-site substrate potential of double-well topology. In this case, the double-well topology of a substrate potential imposes a constraint on the amplitude of the soliton; only that amplitude which is equal to the distance between two minima of the on-site potential is available. This constraint immediately re- 
sults in fixing some value of the supersonic velocity $s$ due to the dependence of the amplitude of the lattice soliton on its velocity. In other words, the presence of some constraints on an anharmonic molecular chain (e.g., fixing its ends) selects from its continuous supersonic spectrum of velocities some discrete values. Such a fixed value of the supersonic velocity has been observed in Ref. [22] for the lattice soliton moving in an on-site potential of the $\phi^{4}$-form. As for the present case, we also have the constraint caused by the zig-zag structure. We expect that in the 3D case, the existence of discrete values of the soliton velocity also holds.

Finally, we notice that the basic set of equations of motion in the continuum limit does not appear to be a simple "complementation" of the Boussinesq equation in its illposed or improved form by an equation for transverse degrees of freedom. In fact, the coupling between the longitudinal and transverse degrees of freedom becomes quite sophisticated. In setting up the continuum version of the discrete equations of motion we have accomplished the procedure similar to deriving the improved form of the 1D
Boussinesq equation. As a result, the coefficient $a_{2}$ which satisfies the inequalities $1 / 4<a_{2}<1$ and depends on the geometry of the system appears in the continuum version of the equations of motion. Note that the ill-posed analog contains another coefficient $a_{1}$ which varies in the broad interval $0<a_{1}<\infty$ (also depending on the geometry of the chain), and therefore the former choice is preferable for analytical studies.

\section{ACKNOWLEDGMENTS}

This work was partially carried out with the financial support from the European Economic Community (EEC) under the INTAS Grant No. 94-3754. One of us (A.V.Z.) would also like to express his gratitude to the MIDIT Center and the Institute of Mathematical Modelling of the Technical University of Denmark for the Guest Professorship and hospitality. Stimulating and useful discussions with T.C. Bountis, D.W. McLaughlin, and A.C. Scott are gratefully acknowledged.
[1] M.D. Kruskal and N.J. Zabusky, J. Math. Phys. 5, 231 (1964).

[2] N.J. Zabusky, Comp. Phys. Commun. 5, 1 (1973).

[3] M. Toda, Phys. Rep. 18, 1 (1975).

[4] M.A. Collins, Chem. Phys. Lett. 77, 342 (1981).

[5] P. Rosenau, Phys. Lett. A 118, 222 (1986).

[6] J.M. Hyman and P. Rosenau, Phys. Lett. A 124, 287 (1987).

[7] O.H. Olsen, P.S. Lomdahl, and W.C. Kerr, Phys. Lett. A 136, 402 (1989).

[8] P.L. Christiansen, P.S. Lomdahl, and V. Muto, Nonlinearity 4, 477 (1990).

[9] V. Muto, P.S. Lomdahl, and P.L. Christiansen, Phys. Rev. A 42, 7452 (1990).

[10] P.S. Lomdahl, O.H. Olsen, and M.R. Samuelsen, Phys. Lett. A 152, 343 (1991).

[11] S.K. Turitsyn, Phys. Rev. E 47, R796 (1993).

[12] M. Peyrard and A.R. Bishop, Phys. Rev. Lett. 62, 2755 (1989).
[13] M. Peyrard, T. Dauxois, H. Hoyet, and C.R. Willis, Physica D 68, 104 (1993).

[14] T. Dauxois, M. Peyrard, and A.R. Bishop, Phys. Rev. E 47, R44 (1993).

[15] N. Flytzanis, St. Pnevmatikos, and M. Remoissenet, J. Phys. C 18, 4603 (1985).

[16] N. Flytzanis, St. Pnevmatikos, and M. Peyrard, J. Phys. A 22, 783 (1989).

[17] S. Cadet, Phys. Lett. 121, 77 (1987).

[18] A.V. Zolotaryuk and A.V. Savin, Physica D 46, 295 (1990).

[19] A.V. Zolotaryuk, St. Pnevmatikos, and A.V. Savin, Physica D 51, 407 (1991)

[20] S. Yomosa, Phys. Rev. A 32, 1752 (1985).

[21] J. Pouget, S. Aubry, A.R. Bishop, and P.S. Lomdahl, Phys. Rev. B 39, 9500 (1989).

[22] A.M. Kosevich and A.S. Kovalev, Solid State Commun. 12, 763 (1973). 